\title{
Design Method for Optimized Infills in Additive Manufacturing Thermoplastic Components
}

\author{
Luis Borunda ${ }^{1}$, Manuel Ladron de Guevara ${ }^{2}$, Jesus Anaya ${ }^{3}$ \\ ${ }^{1,3}$ Universidad Politécnica de Madrid ${ }^{2}$ Carnegie Mellon University \\ Ilborunda.eco@etsav.cat ${ }^{2}$ manuelr@andrew.cmu.edu \\ 3jesus.anaya@upm.es
}

The following article extends and tests computational methodologies of design to consider Finite Element Analysis in the creation of optimized infill structures based on regular and semi-regular patterns that comply with the geometrical constraints of deposition. The Stress-Deformation relationship manifested in Finite Element Analysis is structured in order to influence the geometrical arrangement of the complex spatial infill. The research presents and discusses a program of performance informed infill design, and validates the generalizability of a method of internalizing and automating Finite Element Method (FEM) processing in Fused Deposition Modeling (FDM) workflows, and tests manufacturability of the methods through its ability to handle the FDM process constraints of FEM influenced intricate geometries.

Keywords: Additive Manufacturing, Finite Element Analysis, Fused Deposition Modeling, 3D infill

\section{BACKGROUND}

Additive manufacturing (AM) has seen an exponential growth of application in architecture and construction since the release of several technique patents. Among the most commonly used AM techniques in architecture is Fused Deposition Modeling (FDM), a process based in the numerical control deposition of a plastic material. FDM is an additive manufacturing process based on the principle of stacking layers of a material of plastic consistency through a numerically controlled deposition system. This process builds an object designed previously from a computer-aided design model.

Generally, the understanding of the fabrication process is overridden, since it is an automated process in which a closed mesh or poly-surface is im- ported in a software that makes a stereolithography format file (STL).

One of the principal affordances of Additive Manufacturing to architecture and construction is that it enables cost efficient production of highly complex forms and recent advances in Fabrication Techniques of Architectural Scale Additive Manufacturing (AM) and Spatial Fused Deposition Modeling (Mueller, Im et al. October 2014) with ad hoc computational methodologies of design of structural and functional components provides critical cost (de Soto, AgustíJuan et al. 2018) and time productivity (Bock 2015) innovations in construction automation of highly complex forms, continuously increasing freedom of design and opportunities for global optimization by engineering local differentiations (Hack, Lauer et al. 


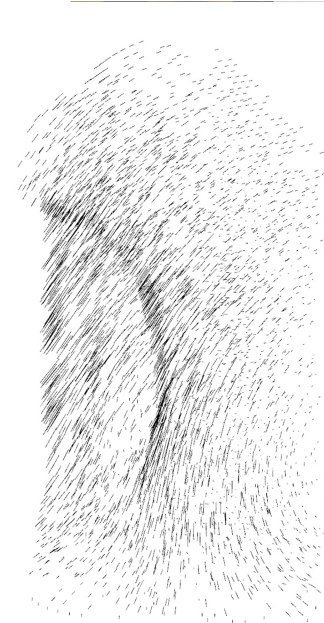

2014).

The 3D printing process distinguishes two major parts, the outer perimeter or boundary of the object, and the interior of the object or infill. Recent efforts focus on topological optimization of the global shape. The parameters of both, the outer layer and the infill can be modified in the STL generator software. However, while we can choose between a limited set of options, infill design is not considered as part of the design process. Customizing the deposition process, as in spatial 3D printing, allows design criteria to be implemented at a material performance level (Ladron de Guevara et al. 2019), further enabling control over the geometry.

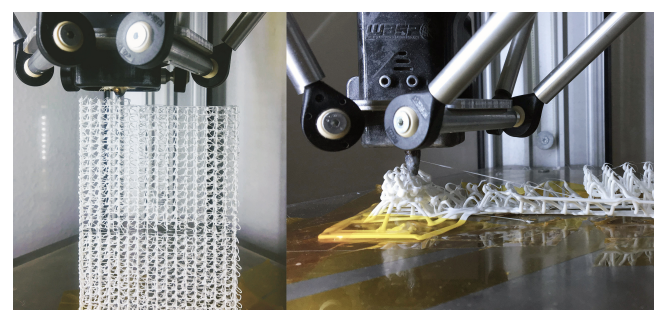

Studies of architecture and large-scale production of structural components exhibit that a competitive time of fabrication and a correct simulation of anisotropic mechanical properties are among the main challenges for future development of the manufacturing process. This research studies the digital design itself, previous to the manufacturing process, to further optimize the material distribution and robotic toolpath production, potentially increasing the performance and time required for production of the processed components, as the principal area of opportunity for significant improvement.

The regular network of threads produced in standard FDM is characterized by having an anisotropic mechanical behavior (Ahn, Montero et al. 2002). Locally influencing the thread deposition during FDM based on FEM simulation results has demonstrated successful transformation of mechanical properties (Gospill, Shindler et al. 2017).

Commercially available Slicing software (Cura, Slic3r) include undifferentiated 3D infills, interesting for their better mechanical properties as compared to continuous vertical extrusion of patterns in $2 \mathrm{D}$ solutions. These geometrical designs present yet some
Figure 1

Custom 3D printing

of cellular

structures

Figure 2

Cloud of threads in multi-resolution spatial 3D printing 
Figure 3

Fiber

discontinuities in processed token with density variation by commercially available slicing software

Figure 4

Unstructured discretized individual values at full Finite Element Analysis resolution divided into 3 isostatic cloud thresholds, low strain, mid strain and high strain challenges for successful FEM simulation, as some of the manufacturability aspects.

Newer developments in slicing software include variations in density and the possibility of manually introducing infill density differentiation by varying the percentage of solid and empty parts within volumetric thresholds defined by solid meshes interior to the global geometry. In this technique the resulting infill are processed and computed as independent volumes, useful for resolution or coloring purposes but fragile in their mechanical performance due to the discontinuity of fibers at volumetric threshold limits (Borunda et al. 2019).

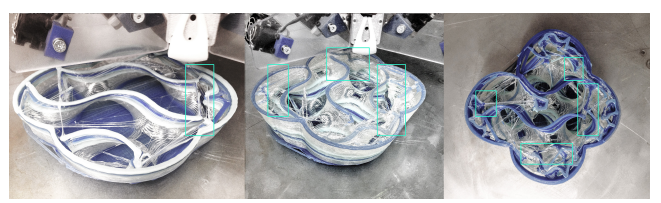

Influencing the infill design using FEM Analysis is a current topic of study. This research builds upon methods for introducing computational workflows that incorporate Finite Element Analysis (FEA) (Chronis, Dubor et al. 2017, Gospill, Shindler et al. 2017) in the design of functional components to apply stress informed AM fabrication techniques (Tam, Mueller 2017) in the fabrication of spatial structures by locally differentiating the deposition of material, specifically tailored for a given application to increase the opportunities for optimization of the mechanical properties of the studied component. A reduced environmental impact can be another significant development in reducing material waste.
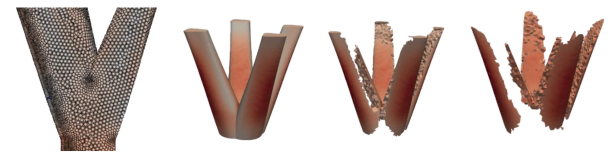

The purpose of this research is to explore where we are heading regarding construction automation by additive manufacturing of informed geometries, particularly exploring the potential improvements to layer by layer Fused Deposition Modeling applications in manufacturing of functionally graded components (Loh et al. 2018), to give insight into digital design workflows that consider impact of data in the geometrical composition and material constitution of form.

\section{METHODS}

The characterization of mechanical behavior in functionally graded components show several potentials in maximizing performance of building component manufactured with FDM techniques. The approach presented in this paper evaluates the potential use of strategies common in bone development (Gibson 1985) of density variation, and a characterization of mechanical performance along the principal stress lines resulting in a foam-like behavior (Gent, Thomas 1956) of the porous cellular solid. AM techniques such as FDM show exceptional capacities of producing very complex structures.

The process of fused deposition requires the serialization deposition process of a continuous robotic toolpath in discretely computed units (Retsin and Jimenez Garcia 2016). A method for automating the infill differentiation that maintains continuity of fibers and density variations based on Finite Element Analysis can potentially produce highly differentiated mechanical properties for functionally graded building components, with no need of changing material. Foam-like infills manufactured with FDM can be successfully adapted in isotropic to anisotropic mechanical behavior by varying the deposition toolpath. By control of differentiated density infill based on FEM stress results and deformation, vectors of displacement may provide stiffness and increase performance.

After important developments in layer by layer principle of manufacturing, such as advances in industrial grade deposition systems and advances in material engineering, the technique is currently being exploited in an increasing amount of architectural projects. Computational methods of utilizing Fi- 
nite Element Method (FEM) analysis to optimize components by engineering the internal material distribution based on complex three dimensional patterns (3D infills) are presented.

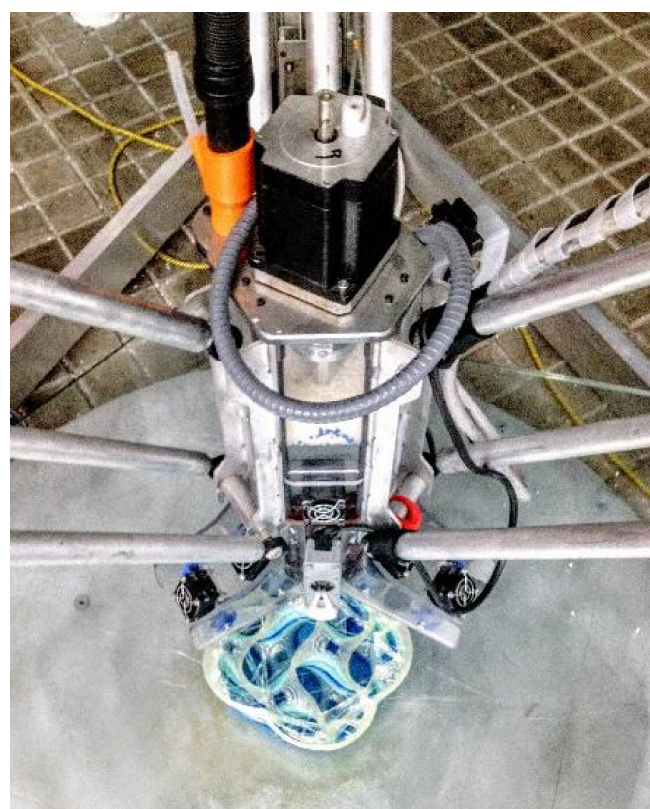

\section{FEM TO GCODE WORKFLOW}

Complex structures are susceptible of Robotic AM for their generalized geometric logic, adaptable to algorithmically design processes by means of discretization of a global geometry in a concatenation of local manufacturable geometries. Each of the computed units, are therefore, directly related to an index corresponding to FEM analyses.

From a holistic point of view, a global geometry is constructed and analyzed with a series of FEM analyses. The result of these analyses informs the construction of a new customized infill that must optimize and improve the rigidity of the piece without any morphological change at the skin of piece. Respectively, the contribution of this research lies in the creation and corroboration of a method for the design and optimization of the infill structure of fused deposition modeled components for the fabrication of digitally designed complex surfaces composed of discrete polymer components.

The characterization of fibrous structures that perform as cellular solids based uniquely on their geometrical difference provides further insight in the use of several types of infills for different density applications.

This research takes a non-structural dented triangular column as a case study and explores the potential of infill variations to accommodate a priori instability to a successful manufacturable column, based on an FEM-informed redesign of the infill pattern that builds on differentiated complexity and rigidity.

We depart from a vertically extruded filletedangle triangular shape and apply some denting force until FEM analyses produce unsatisfactory results. Once the geometry is suitable to be reconfigured with an informed infill pattern, we build an algorithm that identifies localized stressed points and transforms the infill pattern at the stressed areas to elevate its rigidity level.

Custom feedback loop and data links are required to FEM process FDM pieces, a standard workflow is yet to be developed. The generalized process proposed for this research follows:

1. Geometries are modeled in gh-python for Grasshopper, plugin for Rhinoceros software.

2. Geometries are imported to SimScale Cloud based FEM for discretization and analysis.

3. ParaView unstructured data is exported as csv tables through python shell.

4. Tables of data are imported back to Rhinoceros using Python.

5. Custom python components in Grasshopper parse data and create geometrically continuous deposition infill designs as a list of coordinates.

6. The list of coordinates is computed as Gcode commands.
Figure 5

Polyhedral 3D infill with continuous fiber deposition 
Figure 6

Sequence of applying denting force until FEM analyses yields unsatisfactory structural results.

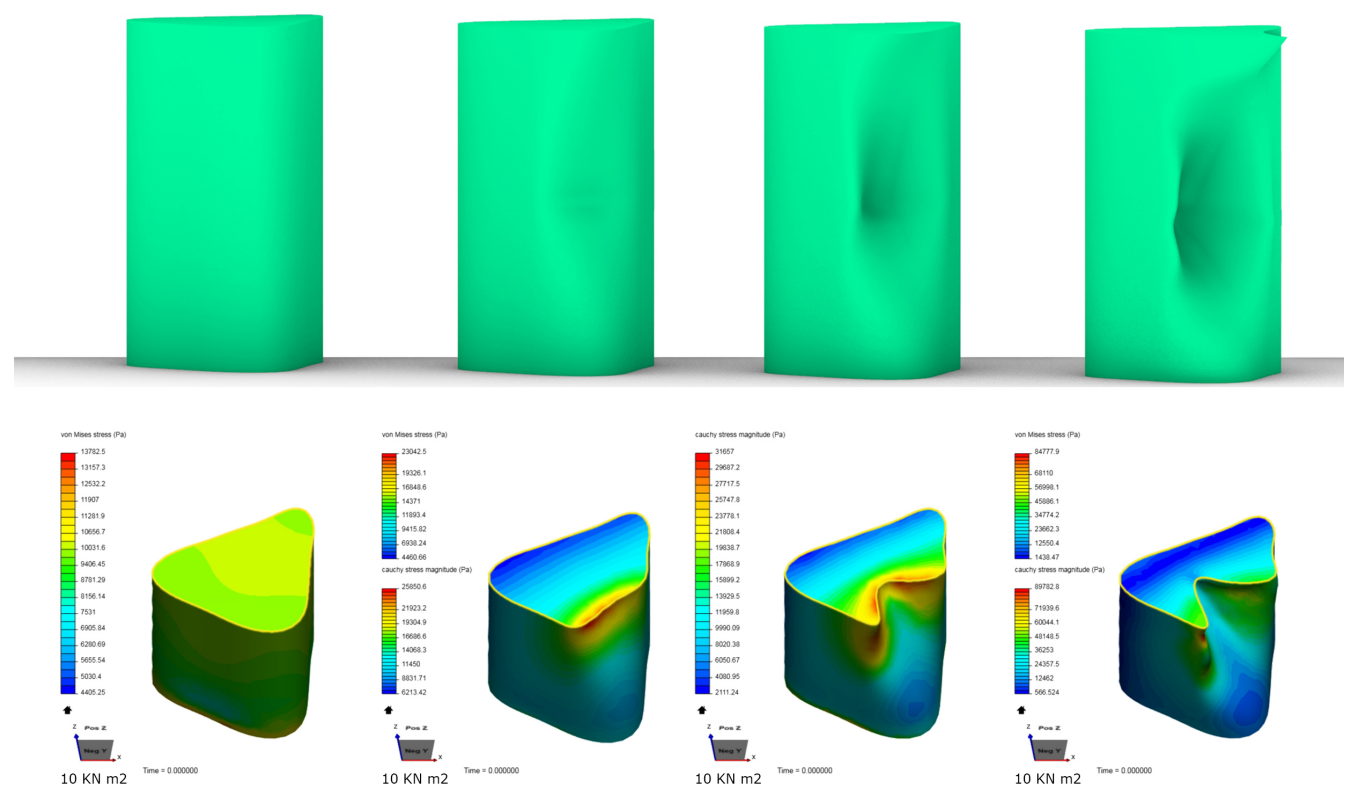

The set of coordinates in a table is transferred to commands for 3D print. The Gcode Commands must review that each point is not self-intersecting and is be structured following a generalized method of deposition. Each point of the list can be categorized in the following:

- Base: for which ventilators are turned off, flow is increased and speed is reduced.

- Knot: a point or line to be fused with previous layer for which ventilators are turned off.

- Thread: a line in space with cooling system on. Speed is reduced.

- End point: stops extrusion and gently lifts nozzle for initiating a no-extrusion traveling movement.
The computational method is tested in dented designs, particularly susceptible of concentrating stress, therefore significantly benefiting from a highly differentiated material distribution and infill layout. FEM influenced FDM is particularly relevant for the production of singular instances of performative and parametric models in digital design due to their intrinsic complexity. A set of parametrically design dented structures (Figure 6) is modeled and fabricated for the purpose of testing variable matrices and infill mechanical performance.

This dented geometry is discretized in external cloud based simulation SimScale. The discretization process hinges on 3D mesh generating system common for Fluid Dynamics simulations. This 3D mesh provides a system for indexing stress values in 

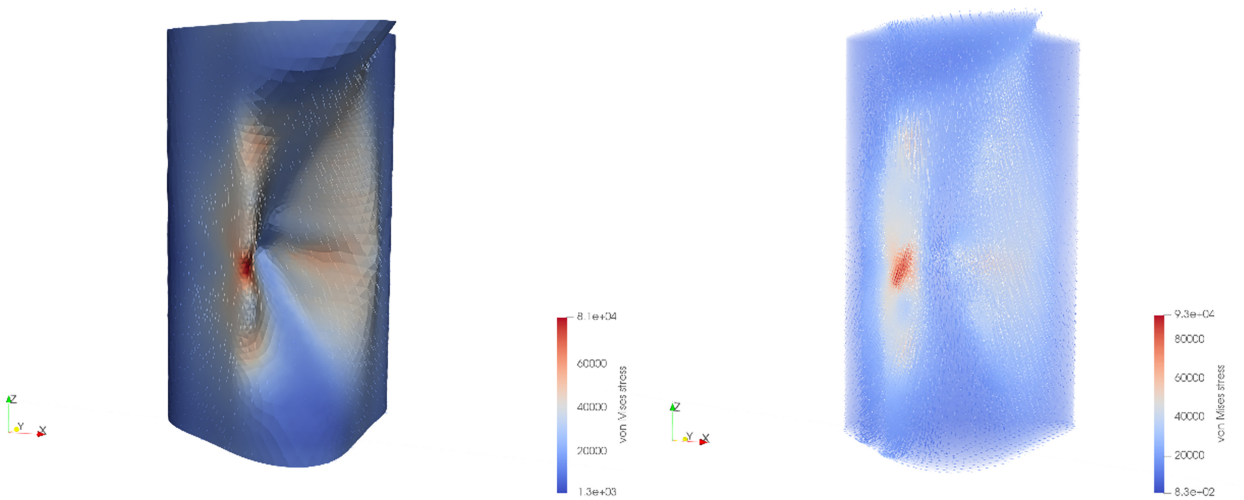

Figure 7

Indexed stress

values in a 3D mesh

structure

3D space (Figure 7) accessible through Visualization Toolkit (VTK).

The design process analyses the studied component, a filleted triangular column by overlying the $3 \mathrm{D}$ surface of the model with its FEM paraview output.

\section{DESIGN METHODOLOGY}

The design method comprises a feedback loop process in which FEA analyses inform a proposed threedimensional infill pattern. Since it is structured based on a three-dimensional grid with different resolutions, a combination between a layer-by-layer approach and a spatial printing technique is required. The infill is no longer a fixed two-dimensional pattern repeated layer by layer. Rather, it assumes a principal role in the design process.

While the perimeter of the object is independent form this process, being naturally designed first, the design of the infill goes into a loop until the FEM analyses meet. An algorithm joins perimeter and infill at each loop iteration, ensuring the unification of both parts. The results obtained from finite element analysis (FEA) engineer anisotropic plastic components by discretely determining infill-like spatial polyhedral geometries. In order to produce bespoke infill patterns, a computational method of data processing based on a graph data structure is required in order to relate two different object classes that manipulate FEM data such as a singular data-point and a singular data-layer, with a class that creates infill patterns.

Initial indexing of cells takes place with the internal discretization process of a global shape. This provides an index to each one of the particles that composes an isostatic cloud. As each cell is composed of geometrical and performance information referenced to one point, it is possible to access this information in the software Paraview, from where a table of results is extracted in csv format. This extraction process is automated in Python, and the abstraction of geometrical and spatial values in a table enables the successful processing of samples in orders of magnitude superior to 100.000 samples with a commercially available processing equipment.

We take unstructured results from stress analysis data sources in isovolumes and isosurfaces in order to locally differentiate the composition of plastic tokens (density, mechanical properties and fidelity) based on different analyses such as associated stress values. This set of samples, compounded by units in the isostatic cloud list of points, stress values and deformation vectors is processed by an algorithm that organizes the data, converting the unstructured FEM 
data to a structured object classes. After applying the underlying structure, an ordering algorithm indexes the isostatic cloud by layers, and outputs it ordered by $Z$ key, that is, first re-structuring by interpretation of values in order of height, as the deposition process requires, then, negotiating in relationship with the global set results and the neighboring results of the specific geometrical design and value of density of the sample.

Internal discrete structures can greatly enhance overall material performance, specifically strength and stiffness are evaluated and improved. We in-
Figure 8

von Mises stress values on studied dented surface
Figure 9

Original pattern vs FEM-informed pattern at localized stress area.
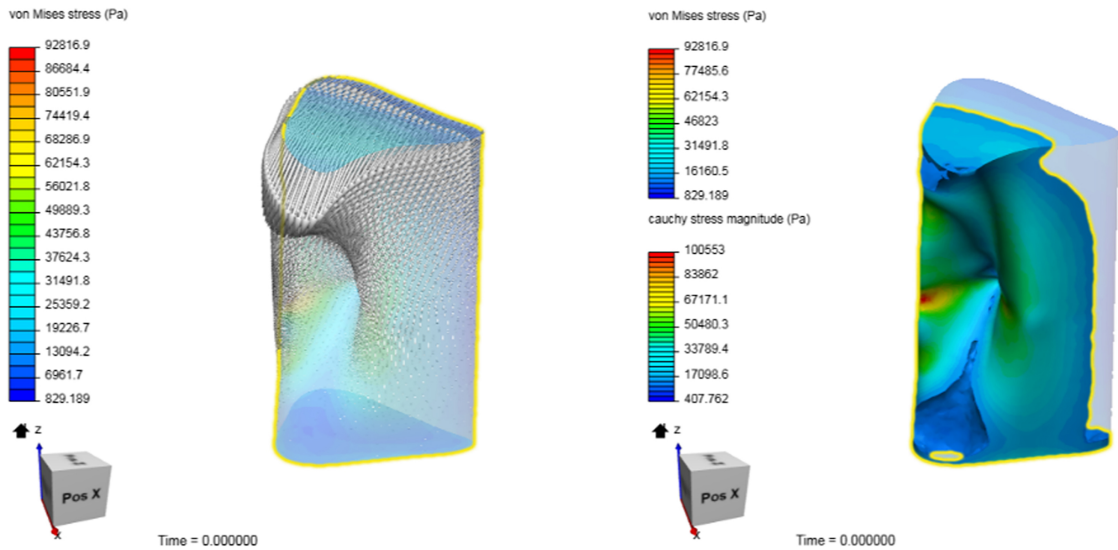

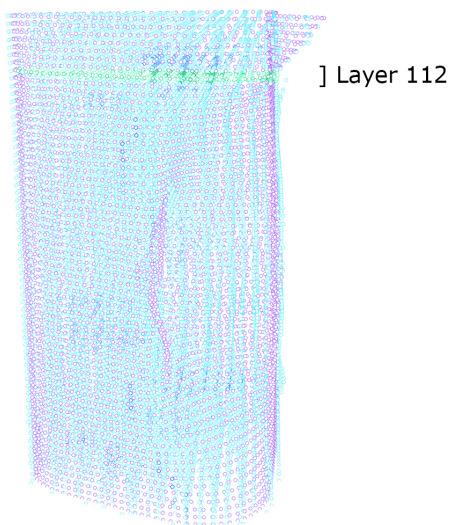

Structured point cloud

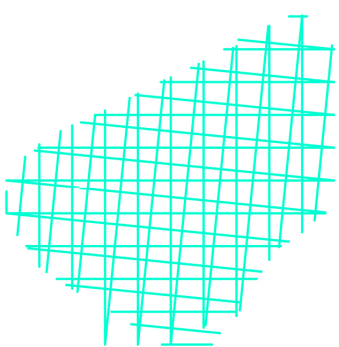

Original Pattern

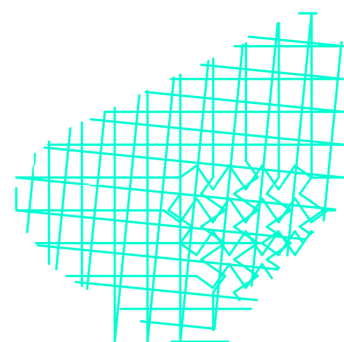

FEM Informed Pattern 


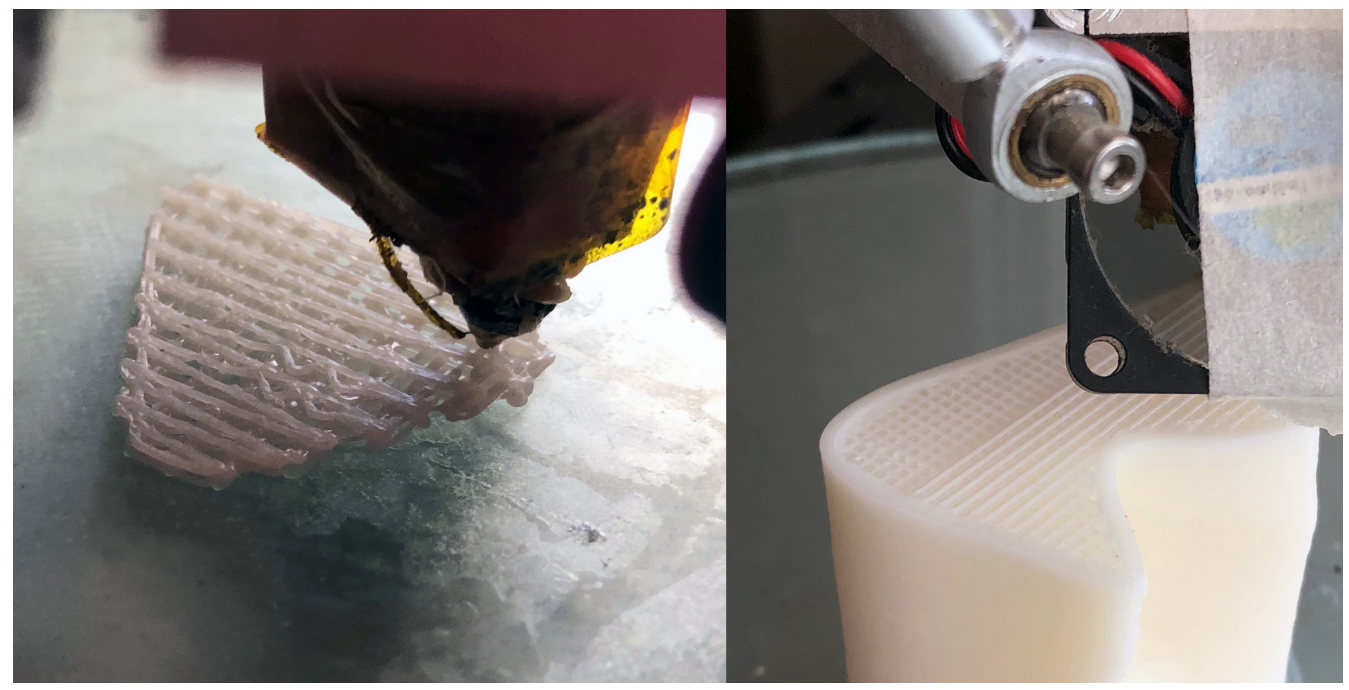

Figure 10

Informed infill layer vs default infill from Gcode exporter software

crease performance while potentially reducing material use and manufacturing time, two of the main constraints in adapting the rapidly growing use of additive manufacturing in larger scale applications.

The design method workflow is applied to robotically augmented additive manufacturing techniques and large scale industrial additive manufacturing techniques. The paper validates the method through testing FEA to FDM processed tokens and standard infill designs at different loading scenarios.

\section{COMPUTATION OF INFORMED INFILL PAT- TERN}

The algorithm creates and indexes a set of points that correspond to discrete areas of horizontal sections. This set of points creates an infill pattern by connecting each point with polylines, in a process similar to what any current default Gcode exporter software does to 3D print a component - namely, Cura, Slic3r, or OctoPrint -. The algorithm then, redesigns the mentioned patterned infill, informed directly by the FEA process. This allows us to create customizable intelligent infill geometries that make localized densi- ties respond to structural analyses.

Computationally, points are organized through an underlying graph data structure in which each point is represented by a vertex, and it is connected with its neighbors by edges. The vertices of the graph are dictionaries of data that contain 3D points in space, and information about their location, the FEM result at this precise location and the quantity and quality of its neighbors. We map the result from the FEM analysis to level of priorities. The more critical the smashed surface is, the higher level it has, and the more neighbors it creates in order to get it optimized until making it pass the FEM analysis again. It is important to say that each index is aware about its neighbors, and its neighbors about their closer ones. What it drives the configuration of the total point cloud is the information residing at each vertex. Based on each neighbor's levels, a particular point may re-adjust its position to satisfy the holistic configuration. 


\section{RESULTS AND DISCUSSION}

Current computational power opens up new design methodologies, shifting the conventional visualfeedback design process in architecture to a more scientific approaches. This paper presents an optimization workflow for structurally unstable surfaces to find a successful solution by informing the infill pattern through FEM analyses. Its contribution lies in presenting an alternative of making successfully stable complex surfaces without the need of modifying the global shape. A general understanding of programming and FEA tools is required, also, combined with the ability of producing an anew gCode. While available gCode generator software is only a tool for quick manufacturing that exists at the end of the design-fabrication process, we understand there is an opportunity of design intelligently by inserting the gCode stage earlier in the overall process.

Methods for translating unstructured data obtained from other Computational Analysis by Finite Elements into geometrical 3D infill data in continuous deposition toolpaths can be implemented, not only to allow for mechanical behavior improvements but to introduce novel information workflows into the design and manufacturing processes.

Even though a new customized infill is created that responds directly to the specific dented surface, this is only one step towards a full customizable infill design. A singular pattern has been developed, demonstrating the close connection between FEA models and FDM. Further research will make a comparison between different design infill approaches, will test their mechanical properties and will produce an outcome to determine the most optimized infill that meets the specificity of the studied piece.

This research explores a previously unexplored territory in architectural application, and offers an alternative to make structural, and therefore, manufacturable to a priori non-suitable geometries.

\section{REFERENCES}

Ahn, SH, Montero, M, Odell, D, Roundy, S and Wright, PK 2002, 'Anisotropic material properties of fused deposition modeling ABS', Rapid Prototyping Journal, 8(4), pp. 248-257

Bock, T 2015, 'The future of construction automation: Technological disruption and the upcoming ubiquity of robotics', Automation in Construction, 59, pp. 113-121

Borunda, L, Ladron de Guevara, M, Anaya, J and Pugliese, G 2019 'Additive Manufacturing Building Components', 4 International Conference on Technological Innovation in Building (CITE), Madrid

Chronis, A, Dubor, A, Cabay, E and Roudsarim, MS 2017 'Integration of CFD in Computational Design. An evaluation of the current state of the art', International Conference on Education and Research in Computer Aided Architectural Design in Europe (eCAADe) 35, Rome

Gent, AN and Thomas, AG 1959, 'The deformation of foamed elastic materials', Journal of Applied Polymer Science, 1(1), pp. 107-113

Gibson, L 1985, 'The mechanical behaviour of cancellous bone', Journal of Biomechanics, 18(5), pp. 317-328

Gospill, JA, Shindler, J and Hicks, BJ 2018, 'Using finite element analysis to influence the infill design of fuseddeposition modelled parts', Progress in Additive Manufacturing, 3, pp. 145-163

Ladron de Guevara, M, Borunda, L and Krishnamurti, R 2019 'Multi-resolution Design Methodology Based on Discrete Models', Springer Nature Singapore Pte Ltd. 2019. J.-H. Lee (Ed.): CAAD Futures 2019, CCIS 1028, Daejon, pp. 1-14

Hack, N, Lauer, WV, Gramazio, F and Kohler, M 2014 'Mesh Mould: Differentiation for Enhanced Performance', Proceedings of the 19th International Conference on Computer-Aided Architectural Design Research in Asia (CAADRIA 2014), Kyoto, pp. 139-148

Loh, GH, Pei, E, Harrison, D and Monzón, MD 2018, 'An overview of functionally graded additive manufacturing', Additive Manufacturing, 23, pp. 34-44

Mueller, S, Im, S, Gurevich, S, Teibrich, A, Pfisterer, L, Guimbretiere, F and Baudisch, P October 2014 'WirePrint: 3D printed previews for fast prototyping', Proceedings of the 27th annual ACM symposium on User interface software and technology, Honolulu, pp. 273-280

Retsin, G and Garcia, MJ 2016 'Discrete Computational Methods for Robotic Additive Manufacturing: Combinatorial Toolpaths', ACADIA 2016, Ann Arbor, 
Michigan, pp. 332-341

Soto, BGd, Agustí-Juan, I, Hunhevicz, J, Joss, S, Graser, K, Habert, G and Adey, BT 2018, 'Productivity of digital fabrication in construction: Cost and time analysis of a robotically built wall', Automation in Construction, 92, pp. 297-311

Tam, KMM and Mueller, CT 2017, 'Additive Manufacturing Along Principal Stress Lines', 3D Printing and Additive Manufacturing, 4(2), pp. 63-81 